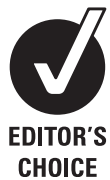

CHOICE

\title{
Imaging in pulmonary hypertension, part 1: clinical perspectives, classification, imaging techniques and imaging algorithm
}

\author{
Caroline McCann, ${ }^{1}$ Deepa Gopalan, ${ }^{1}$ Karen Sheares, ${ }^{2}$ Nicholas Screaton ${ }^{1}$
}

'Department of Radiology, Papworth Hospital, Cambridge, UK

${ }^{2}$ Pulmonary Vascular Diseases Unit, Papworth Hospital, Cambridge, UK

\section{Correspondence to}

Dr Deepa Gopalan, Department of Radiology, Papworth Hospital, Papworth Everard, Cambridge CB23 3RE, UK; deepa. gopalan@btopenworld.com

This article is the first in a series of three on imaging in pulmonary hypertension.

Received 3 July 2011 Accepted 3 December 2011

Published Online First

21 January 2012

\section{ABSTRACT}

Pulmonary arterial hypertension (PAH) is an uncommon condition associated with significant morbidity and mortality. It has diverse aetiology with differing clinical presentations, imaging features and treatments that range from surgical treatment of proximal chronic thromboembolic disease to targeted medical therapies in small vessel disease. Current classification of pulmonary hypertension $(\mathrm{PH})$ is clinically based and groups diseases with similar pathophysiological mechanisms and therapeutic approaches. Groupings include conditions characterised by diffuse small vessel diseases such as idiopathic PAH, PH secondary to chronic hypoxic lung disease, left sided cardiac disease, chronic large vessel obstruction such as chronic thromboembolic disease and a miscellaneous group of diseases. The physiological manifestation of all of these diseases is increased pulmonary vascular resistance and PAH and while clinical features may provide a clue to diagnosis imaging plays a fundamental role in establishing a precise diagnosis and therefore guides therapy. A broad range of imaging modalities is available for the patient with suspected $\mathrm{PH}$ including chest radiograph, echocardiography, ventilation/perfusion scintigraphy, catheter pulmonary angiography as well as cross-sectional CT and MRI. Each modality has its strengths and limitations and different techniques may be used at different stages of diagnostic investigation and frequently complement each other. For example, while MRI and echocardiography permit cardiac structural and functional assessment, CT pulmonary angiography provides exquisite morphological information about the proximal pulmonary vasculature and lung parenchyma but little functional information. Modern cross-sectional imaging techniques (CT and MRI) hold the promise of a comprehensive evaluation of the heart, circulation and lung parenchyma in $\mathrm{PH}$. The authors present a multimodality-imaging algorithm for the investigation of patients with suspected $\mathrm{PH}$ though it is acknowledged that there is some variation in practice depending on availability of resources and expertise.

\section{INTRODUCTION}

Pulmonary hypertension (PH) is a haemodynamic and pathophysiological condition defined as an increase in mean pulmonary arterial pressure (MPAP) of $25 \mathrm{~mm} \mathrm{Hg}$ or more at rest as assessed by right heart catheterisation. ${ }^{1}$ Pulmonary arterial hypertension (PAH) is characterised by the presence of precapillary $\mathrm{PH}$ in the absence of causes of postcapillary $\mathrm{PH}$ such as left heart disease with a pulmonary capillary wedge pressure (or left ventricular end diastolic pressure) of $15 \mathrm{~mm} \mathrm{Hg}$ or less. Recent systematic reviews suggest that normal MPAP at rest is in fact $14 \pm 3 \mathrm{~mm} \mathrm{Hg}$ with $20 \mathrm{~mm} \mathrm{Hg}$ being the upper limit of normal. ${ }^{2}{ }^{3}$ The significance of an MPAP between 21 and $24 \mathrm{~mm} \mathrm{Hg}$ is unclear. It has also been demonstrated that normal individuals can achieve MPAPs of much more than $30 \mathrm{~mm} \mathrm{Hg}$ on exercise, particularly older individuals. ${ }^{4}$ Therefore, absolute values for pulmonary vascular resistance and a pressure threshold for exercise have been removed from the current definition. ${ }^{5}$

$\mathrm{PH}$ is a significant cause of mortality and morbidity but commonly presents with nonspecific clinical signs and symptoms. Progressive disease leads to right ventricular failure and ultimately death. Causes of $\mathrm{PH}$ are diverse. The most common causes are secondary to either chronic left sided cardiac or pulmonary disease. Disease primarily characterised by narrowing of the small vessels results in increased pulmonary vascular resistance and $\mathrm{PH}$; increased understanding of the underlying biology of these diseases has led to a range of effective novel targeted therapies. Obstruction of the large vessels particularly by chronic thromboembolic disease is increasingly recognised as a cause of $\mathrm{PH}$. Patients in whom the distribution of disease lies predominantly in the proximal vasculature may be suitable for surgical pulmonary endarterectomy with the potential for symptomatic and physiological cure.

It is clear that an accurate and early diagnosis of $\mathrm{PH}$ and its cause are important to guide therapeutic decision-making. Imaging plays a fundamental role. We describe the clinical signs and symptoms of $\mathrm{PH}$, explain the current $\mathrm{PH}$ classification and illustrate various imaging techniques that are available to investigate this condition. The strengths and weaknesses of each of these techniques will also be highlighted.

\section{CLINICAL PRESENTATION}

Presenting symptoms are often non-specific which usually leads to a delay in diagnosis averaging about 2 years or indeed misdiagnosis. ${ }^{6}$ Symptoms are initially those of exertional dyspnoea, but then as the right ventricle (RV) heart becomes increasingly compromised, patients can suffer from reduced exercise tolerance, fatigue, exertional chest pain and syncope. The physical signs include jugular vein distension, left parasternal heave, an accentuated pulmonary component of the second heart sound, a pansystolic murmur of tricuspid regurgitation, hepatomegaly, ascites and pedal oedema. Depending on the cause of the $\mathrm{PAH}$, there may be 
other associated signs such as Raynaud's phenomenon in connective tissue disease. The prognosis in PAH depends on several factors, notably the underlying cause and severity, but any time lag to diagnosis will also play a part. Ultimately, the magnitude of the increase in pulmonary vascular resistance and the functional reserve of the RV are key prognostic determinants, with the most marked impairment being associated with the worst prognosis. The median survival with untreated idiopathic PAH (IPAH) is estimated at approximately 2.8 years and 5 -year survival $34 \%{ }^{7}$

\section{Box 1 Updated clinical classification of pulmonary}

\section{hypertension (Dana Point, 2008)}

1 Pulmonary arterial hypertension (PAH)

1.1 Idiopathic PAH

1.2 Heritable

\subsubsection{BMPR2}

1.2.2 ALK1, endoglin (with or without hereditary haemorrhagic telangiectasia)

\subsubsection{Unknown}

1.3 Drug-induced and toxin-induced

1.4 Associated with:

1.4.1 Connective tissue diseases

1.4.2 HIV infection

1.4.3 Portal hypertension

1.4.4 Congenital heart diseases

1.4.5 Schistosomiasis

1.4.6 Chronic haemolytic anaemia

1.5 Persistent pulmonary hypertension of the newborn

1 ' Pulmonary veno-occlusive disease and/or pulmonary capillary haemangiomatosis

2 Pulmonary hypertension owing to left heart disease

2.1 Systolic dysfunction

2.2 Diastolic dysfunction

2.3 Valvular disease

3 Pulmonary hypertension owing to lung diseases and/or hypoxia

3.1 Chronic obstructive pulmonary disease

3.2 Interstitial lung disease

3.3 Other pulmonary diseases with mixed restrictive and obstructive pattern

3.4 Sleep-disordered breathing

3.5 Alveolar hypoventilation disorders

3.6 Chronic exposure to high altitude

3.7 Developmental abnormalities

4 Chronic thromboembolic pulmonary hypertension

5 Pulmonary hypertension with unclear multifactorial mechanisms

5.1 Haematologic disorders: myeloproliferative disorders, splenectomy

5.2 Systemic disorders: sarcoidosis, pulmonary Langerhans cell histiocytosis, lymphangioleiomyomatosis, neurofibromatosis, vasculitis

5.3 Metabolic disorders: glycogen storage disease, Gaucher disease, thyroid disorders

5.4 Others: tumorous obstruction, fibrosing mediastinitis, chronic renal failure on dialysis

Main modifications to the previous Venice classification are in given in bold.

ALK1, activin receptor-like kinase type 1; BMPR2, bone morphogenetic protein receptor type 2 .

\section{CLASSIFICATION OF PH}

Classification of any disease, whether by anatomical site, pathophysiological mechanism or clinical presentation, aids in making the diagnosis and treatment. There have been multiple changes to the PAH classification over the last few decades. The initial 1973 version by $\mathrm{WHO},{ }^{1}$ whereby PAH had only two categories, primary or secondary (depending on the presence or absence of any identifiable cause or risk factor), has long since been refined. Since then, there have been three World Symposia on PAH in: (1) Evian, France (1998), ${ }^{8}$ (2) Venice, Italy (2003) ${ }^{9}$ and most recently (3) Dana Point, California (2008). ${ }^{10}$ The current classification (box 1) maintains the general organisation of previous classifications but incorporates additional information published over the last 5 years and also provides more clarity on some specific points. The general aim is to group according to similar aetiology and therapeutic options.

The most recent modifications in the Dana Point Classification include: replacement of the term familial PAH by heritable PAH because specific gene mutations have been identified in sporadic cases with no family history. Heritable forms of PAH include IPAH with germline mutations and familial cases with or without identified germline mutations ${ }^{11-13}$; Pulmonary venoocclusive disease and pulmonary capillary haemangiomatosis have been included in a new subgroup of Group 1, that is, Group 1 ', the reason being that although they are distinct diseases in themselves, they do share similarities with IPAH and should not be completely separated from the PAH group $;{ }^{5}{ }^{10}$ schistosomiasis and chronic haemolytic anaemia appear as separate entities in the subgroup of PAH associated with identified diseases. The literature indicates that these conditions can have similar histological findings to IPAH, including plexiform lesions. ${ }^{14} 15$

\section{PH: IMAGING TECHNIOUES}

In addition to laboratory blood tests, ECG, pulmonary function tests and functional assessment, imaging plays a vital role in the preparation for investigation of $\mathrm{PH}$. This includes the detection, characterisation and monitoring of $\mathrm{PH}$. Imaging tests can be

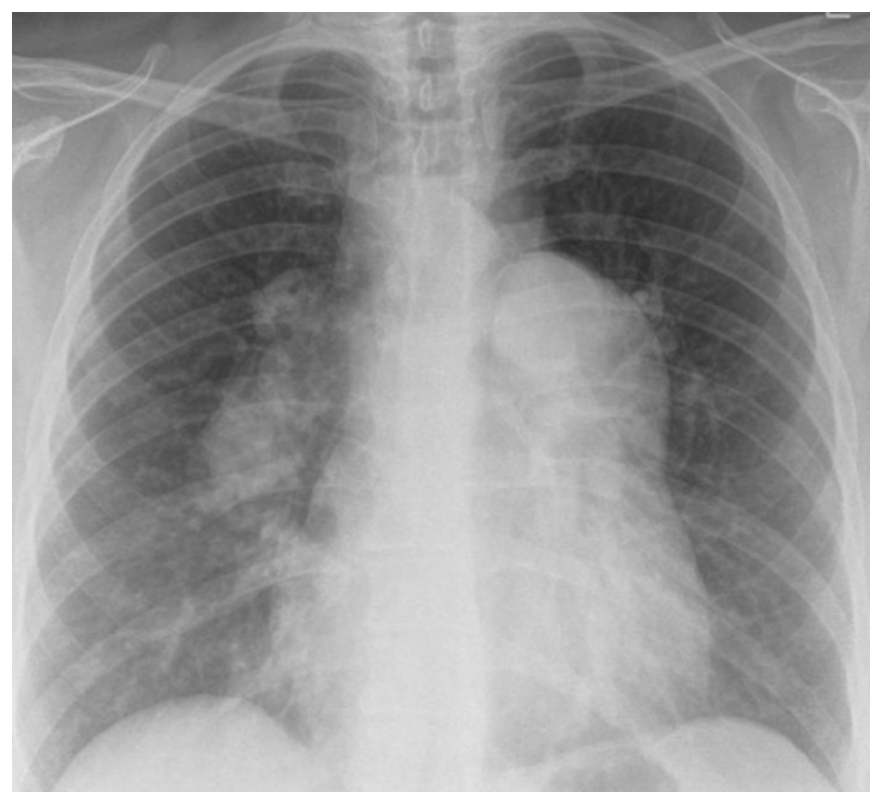

Figure 1 Chest radiograph in a 45-year-old female patient with idiopathic pulmonary arterial hypertension shows massively dilated proximal pulmonary vasculature and peripheral attenuation of vessels. 


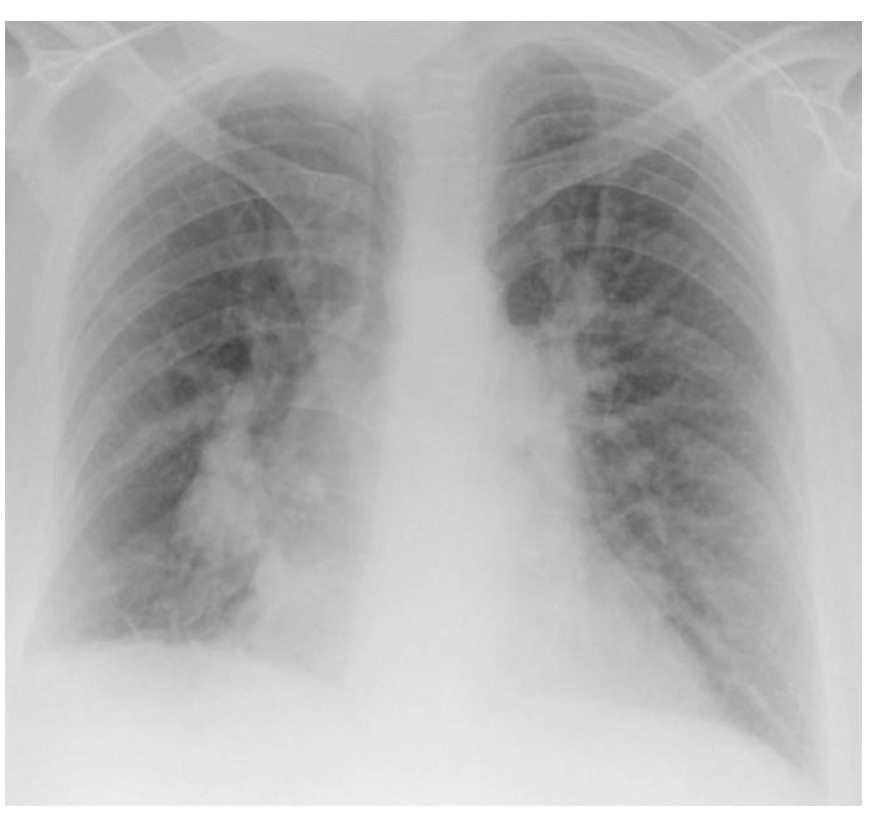

Figure 2 Chest radiograph in a 25-year-old female patient with Down's syndrome and atrial septal defect. There is moderate cardiomegaly with prominent proximal pulmonary arteries and plethoric lungs.

invasive or non-invasive, each having their own strengths and weaknesses. The manifestations of $\mathrm{PH}$ in general will be described for each of the different modalities with a suggested imaging algorithm. Disease specific features will be discussed in subsequent articles.

\section{CHEST RADIOGRAPHY}

The chest radiograph is usually the initial imaging study to be performed in patients with unexplained cardiorespiratory symptoms. Ninety per cent of patients with IPAH have an abnormal radiograph at the time of diagnosis. ${ }^{16}$ The features of $\mathrm{PH}$ on the chest radiograph include cardiomegaly, right atrial and right ventricular dilatation, and enlarged central pulmonary arteries (figure 1). Pulmonary vessels in PAH are typically dilated centrally and attenuated peripherally ('pruned'). However, in the presence of a significant left to right shunt the peripheral pulmonary arteries may appear 'plethoric' (figure 2). Central pulmonary arterial diameter can be used as a guide to the presence of $\mathrm{PH}$. On the right side, the interlobar artery at the hilum should measure no more than $15 \mathrm{~mm}$ in diameter in women and $16 \mathrm{~mm}$ in men. ${ }^{17}$ The left sided vessels are seen better on the lateral projection and should measure no more than $18 \mathrm{~mm}$ from the posterior margin of the artery to the upper limit of the left upper lobe bronchus. ${ }^{18}$ Pulmonary arterial calcification can occasionally be seen in PAH but chest radiography is relatively insensitive. The appearance is due to calcific atherosclerosis from severe and prolonged $\mathrm{PAH}$, usually from shunts with high vascular resistance. ${ }^{19}$ The chest radiograph is also helpful in excluding pleuroparenchymal lung diseases, although in general in these diseases the degree of PH does not correlate well with the severity of radiographic findings.

\section{ECHOCARDIOGRAPHY}

Echocardiography allows structural and functional assessment of the cardiac chambers, and the estimation of pulmonary artery pressure (PAP). It may identify causes of $\mathrm{PH}$ such as left ventricular dysfunction, valvular disease and intracardiac shunts. It is readily available, inexpensive, non-invasive and is used in the initial diagnostic preparation for $\mathrm{PH}$ and also in the followup. Its limiting factors are operator dependency as well as patient related factors such as tachycardia and obesity.

The estimation of PAP is based on the peak velocity of the jet of tricuspid regurgitation, which is found in more than $90 \%$ of patients with severe PAH. The modified Bernouilli equation describes the relationship of peak velocity in $\mathrm{m} / \mathrm{s}\left(\mathrm{V}_{\max }\right)$ and the peak pressure gradient across the valve in $\mathrm{mm} \mathrm{Hg}(\Delta \mathrm{P})$. The equation is: $\Delta \mathrm{P}=4\left(\mathrm{~V}_{\text {max }}\right)^{2}$. The equation takes right atrial pressure into account, which can be estimated from the diameter and respiratory variation of the inferior vena cava or is often given a value of 5 or $10 \mathrm{~mm} \mathrm{Hg}^{5}$ The effect of age and body mass index should be taken into account. A tricuspid regurgitant velocity of $3.0 \mathrm{~m} / \mathrm{s}$ on echocardiography corresponds to an MPAP greater than $30 \mathrm{~mm} \mathrm{Hg}$ (figure 3).

Although in general these measurements do correlate with invasive measurements of right ventricular pressures, Dopplerderived pressure estimation may be inaccurate in an individual patient. Underestimation and overestimation of PA systolic pressure are common in patients with severe tricuspid regurgitation. In addition, echocardiography is notoriously inaccurate when tricuspid regurgitation is only mild. The use of contrast echocardiography does significantly increase Doppler signal, allowing more accurate measurement of peak tricuspid regurgitation velocity. 5

Other entities that can be seen on echocardiography are rightsided chamber dilatation, right ventricular hypertrophy, paradoxical septal motion, increased pulmonary regurgitation, short
Figure 3 Four-chamber view from a transthoracic echocardiogram in a 29 year-old female patient. (A) Right heart chamber dilatation and right ventricular hypertrophy (arrow). (B) Tricuspid regurgitation with a retrograde jet flowing into the right atrium on the colour Doppler (arrow). Regurgitant velocity was $5.3 \mathrm{~m} / \mathrm{s}$. LV, left ventricle; $\mathrm{RA}$, right atrium; $\mathrm{RV}$, right ventricle.
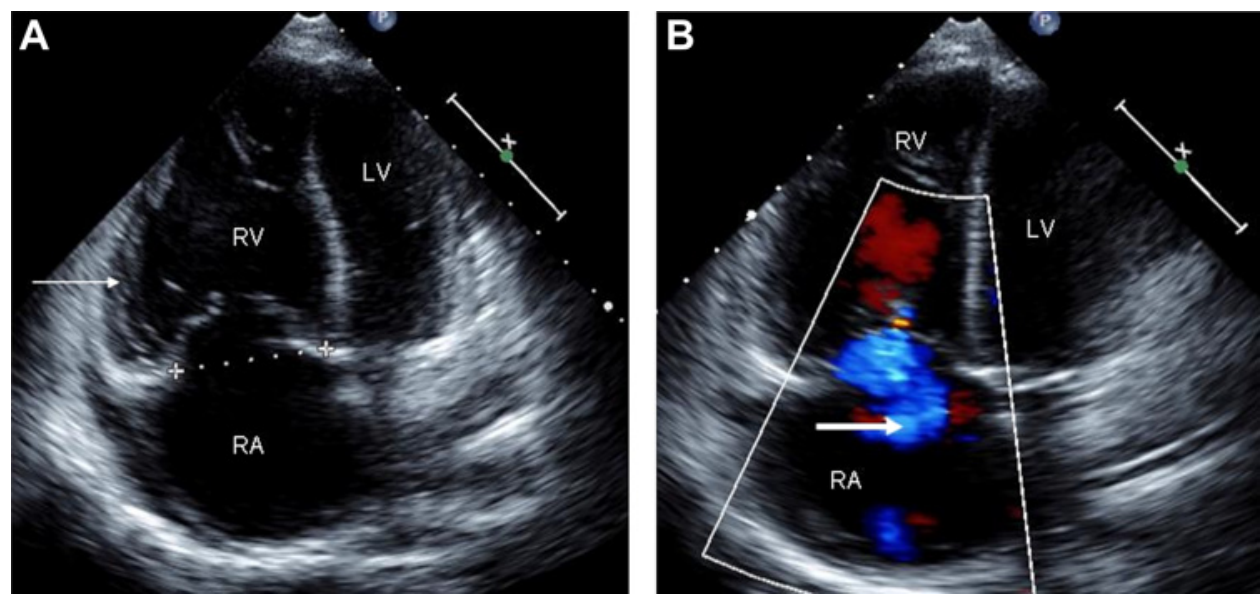

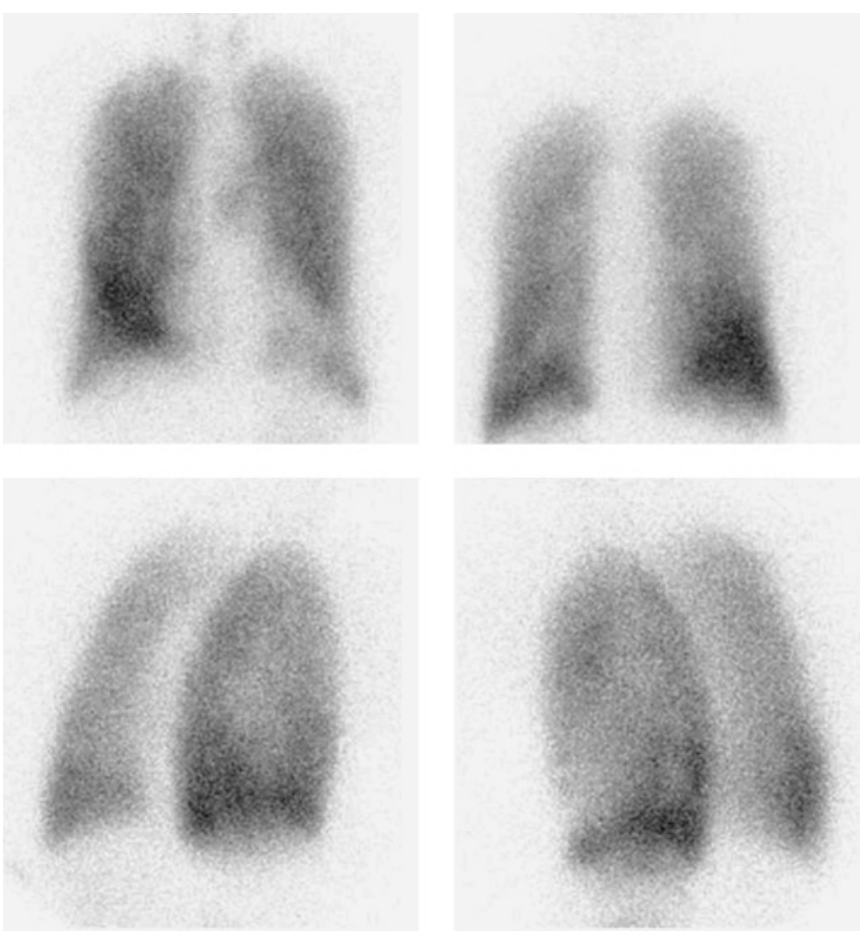

Figure 4 Perfusion scintigraphy in a 45-year-old female patient with idiopathic pulmonary arterial hypertension. The four images show the perfusion series with heterogenous perfusion.

acceleration time of the RV into the pulmonary artery, pericardial effusion and a dilated pulmonary artery.

The feasibility of exercise Doppler-echocardiography in identifying PAH only on exercise is questionable due to the lack of prospective confirmatory data. ${ }^{20}$ Trans-oesophageal echocardiography can also be performed and is particularly useful in identifying shunts, assessing valvular structures and other lesions such as atrial myxomas.

\section{NUCLEAR MEDICINE}

Ventilation/perfusion (V/O) scintigram is mainly performed to identify potentially treatable CTEPH and differentiate it from other causes of PAH. In non-thromboembolic causes of $\mathrm{PAH}$, $\mathrm{V} / \mathrm{O}$ may be normal or non-specifically abnormal showing mismatched non-segmental defects in perfusion giving a 'motheaten' appearance (figure 4). This contrasts with thromboembolic disease where multiple segmental perfusion defects are typically identified with maintenance of normal ventilation, leading to a mismatch of perfusion and ventilation in the affected bronchopulmonary segment (figure 5$)$. V/O has a high sensitivity $(90 \%-100 \%)$ and specificity $(94 \%-100 \%)$ in distinguishing between CTEPH and IPAH, and is more sensitive in this differentiation than $\mathrm{CT}^{21}{ }^{22}$ Its disadvantage is the exposure to ionising radiation and lack of additional anatomical data. Mismatched perfusion defects will also be seen in other causes of $\mathrm{PAH}$ that obstruct/infiltrate the pulmonary arteries, such as extrinsic compression causes, large vessel vasculitis and pulmonary veno-occlusive disease, all of which are described later.

\section{CT}

CT is of great value in the investigation of patients with suspected $\mathrm{PH}$. A combination of contrast-enhanced pulmonary angiography (CTPA) and high resolution CT (HRCT) enable detailed assessment of pulmonary vasculature and the lung parenchyma. Cardiac morphology and tricuspid regurgitation can be evaluated even on ungated studies. Unsuspected intracardiac shunts such as atrial septal defect can be diagnosed even for the first time in adulthood.

On CT, the pulmonary trunk should not measure more than $2.8 \mathrm{~cm}$ at the level of its bifurcation. If it does, this is suggestive of $\mathrm{PH}$ with a sensitivity of $69 \%-87 \%$ and a specificity of
Figure 5 Perfusion scintigraphy in a 60 -year-old male patient with chronic thromboembolic pulmonary hypertension. The four images show the perfusion series with bilateral segmental perfusion defects.
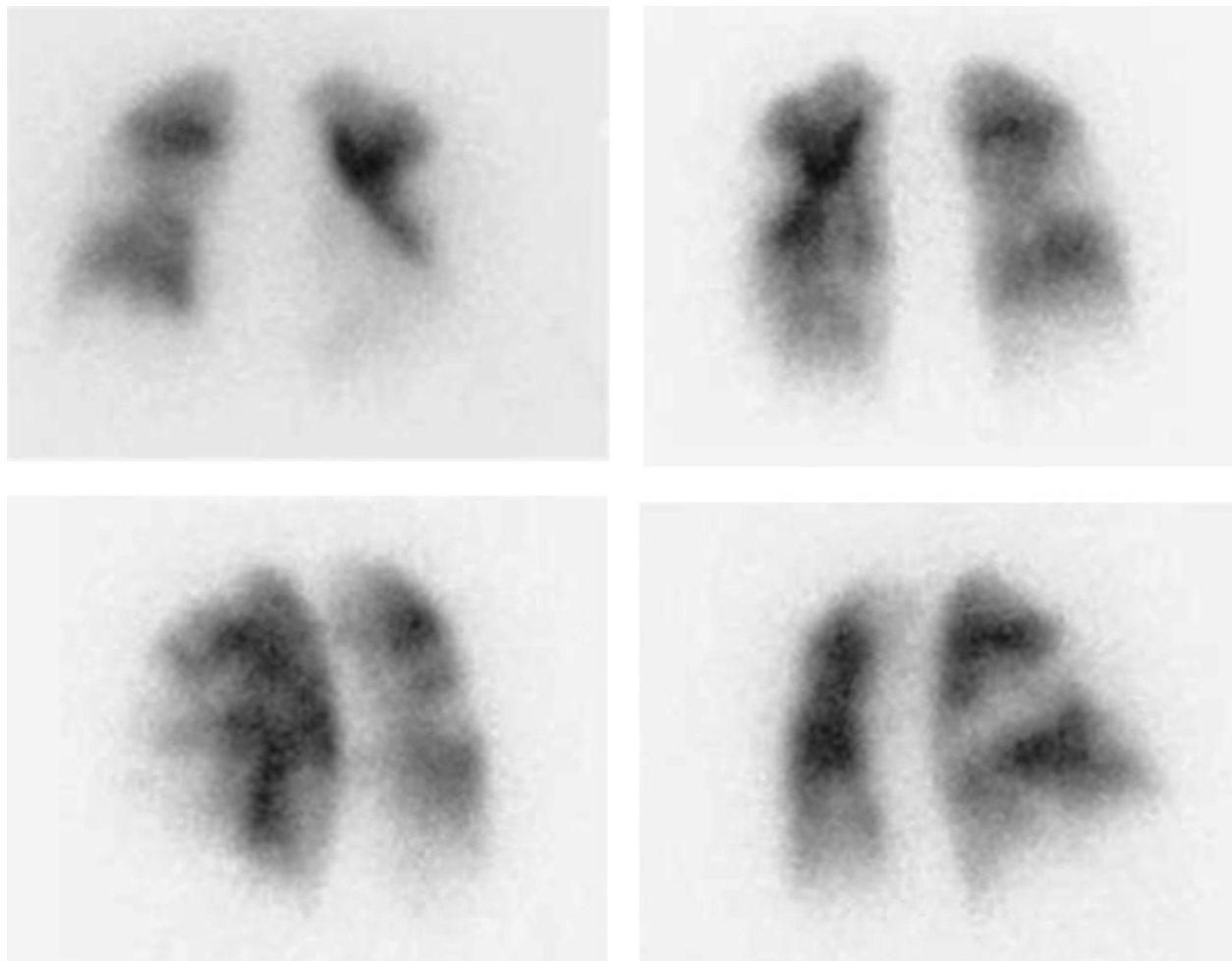

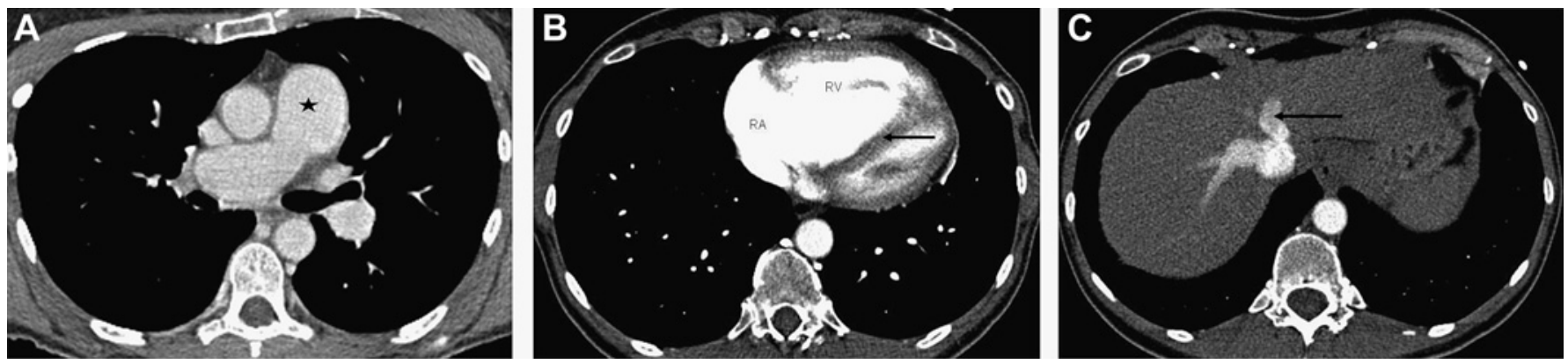

Figure 6 Contrast-enhanced pulmonary angiography in a 55-year-old female patient with idiopathic pulmonary hypertension. (A) An enlarged main pulmonary trunk (star); it measures $3.3 \mathrm{~cm}$ compared with the ascending aorta which measures $2.4 \mathrm{~cm}$ at the same level. (B) Right ventricular transverse diameter is greater than the left ventricular with septal flattening (arrow). RA, right atrium; RV, right ventricle. (C) Reflux of contrast into the hepatic veins in keeping with raised right heart pressure and tricuspid regurgitation (arrow).

$89 \%-100 \%{ }^{23}$ Alternatively, if the pulmonary trunk diameter exceeds that of the corresponding aorta, then $\mathrm{PH}$ must be suspected (figure 6). This method correlates well with MPAP and has a specificity of $92 \%$ and a positive predictive value of $93 \%$. However, its sensitivity is somewhat lower at $70 \%$ with a low negative predictive value of $44 \%{ }^{24}$ This is due to unfolding and dilatation of the aorta particularly in older patients. A recent study attempting to refine this sign with more precise methods in measuring the main pulmonary artery (ie, obtaining the crosssectional area) was not helpful. ${ }^{25}$ An interesting study by Devaraj et al showed that PA dilatation could occur in the absence of $\mathrm{PH}$ in patients with pulmonary fibrosis. ${ }^{26}$ Some studies have explored the more peripheral pulmonary vasculature in $\mathrm{PH}$. In normal subjects, the diameter of the arteries within the lungs should be less than or equal to that of the neighbouring bronchus, while in patients with $\mathrm{PH}$, increased pressure can lead to dilatation of these vessels. An increase in the segmental artery to bronchus ratio in at least three lobes has been shown to be a reliable indicator of $\mathrm{PH}^{25}{ }^{27}$ Devaraj et al showed that segmental arterial size is a reliable marker of MPAP. ${ }^{25}$ However, there may also be $\mathrm{PH}$ related cardiovascular effects that can be easily appreciated on CT including dilatation of the right heart chambers, right ventricular hypertrophy and paradoxical bulging of the interventricular septum (septal bounce) which may result in left ventricular dysfunction. In severe right ventricular failure, there may be ascites, hepatic congestion and peripheral oedema. Reflux of contrast medium into the inferior vena cava/hepatic veins suggests tricuspid regurgitation (figure 7). ${ }^{28}$ Intracardiac shunts and anomalous venous drainage can also be demonstrated (figure 8). As ungated thoracic CT is not acquired at a specific phase of the cardiac cycle and transverse images are not a standard cardiac plane used in echocardiography and MRI, chamber measurements between the modalities may differ. The literature has studies that do and do not show such differences. ${ }^{29-31}$ There are validated figures for CT axial images, the upper limits for the right atrium and ventricle being $35 \mathrm{~mm}$ and $45 \mathrm{~mm}$, respectively. ${ }^{32}$

HRCT facilitates the diagnosis of interstitial lung disease and emphysema. Additional findings of lymphadenopathy and pleural abnormalities can also be readily demonstrated depending on the cause of the $\mathrm{PH}$.

\section{MRI}

Cardiac magnetic resonance (CMR) imaging is a non-invasive three-dimensional cross-sectional technique that allows detailed assessment of cardiac anatomy and function including shunt quantification. The lack of ionising radiation makes MRI advantageous, particularly in the serial follow-up. Disadvantages include the normal contraindications to magnetic resonance such as pacemaker devices, foreign metallic objects and claustrophobia. Protracted scan time, breath-hold limitation and arrhythmias are other limitations.

The gradient-echo or steady state free precession pulse sequences can be used to construct a cine series of 15-20 frames across the full cardiac cycle. This technique allows wall motion abnormalities, ventricular volumes, ejection fraction and
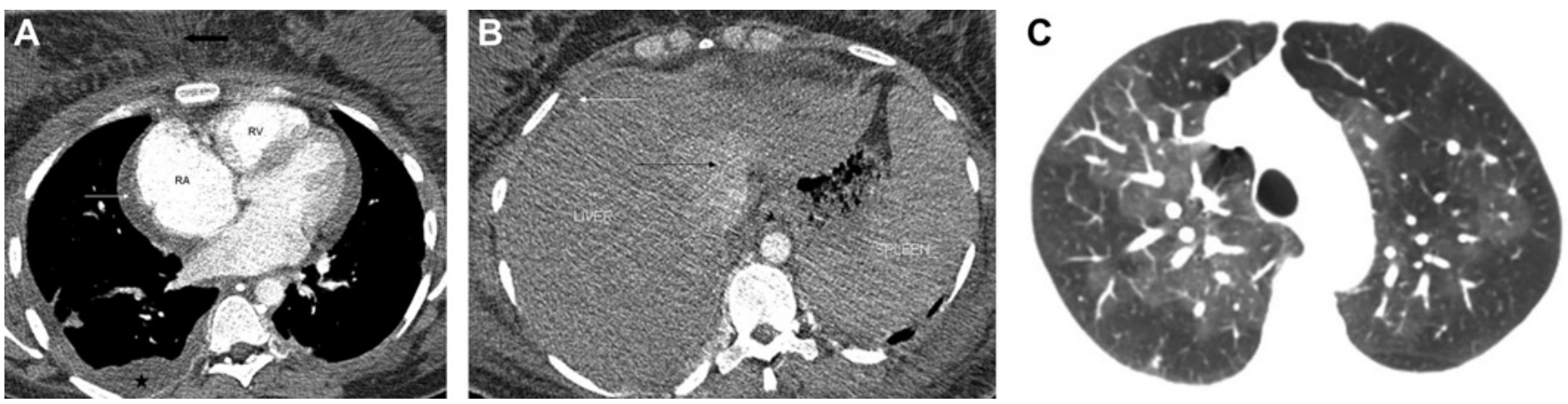

Figure 7 Contrast-enhanced pulmonary angiography in a 36-year-old female patient with chronic thromboembolic pulmonary hypertension with severe pulmonary hypertension and right heart failure. (A) There is right heart chamber dilatation, a pericardial effusion (white arrow), right pleural effusion (star) and subcutaneous oedema in the soft tissues (black block arrow). RA, right atrium; RV, right ventricle. (B) Organomegaly, ascites (white arrow) and contrast reflux into the hepatic veins (black arrow). (C) Lung windows reveal a mosaic perfusion pattern. The pulmonary artery diameter is reduced within the areas of reduced attenuation due to hypoxic vasoconstriction. 

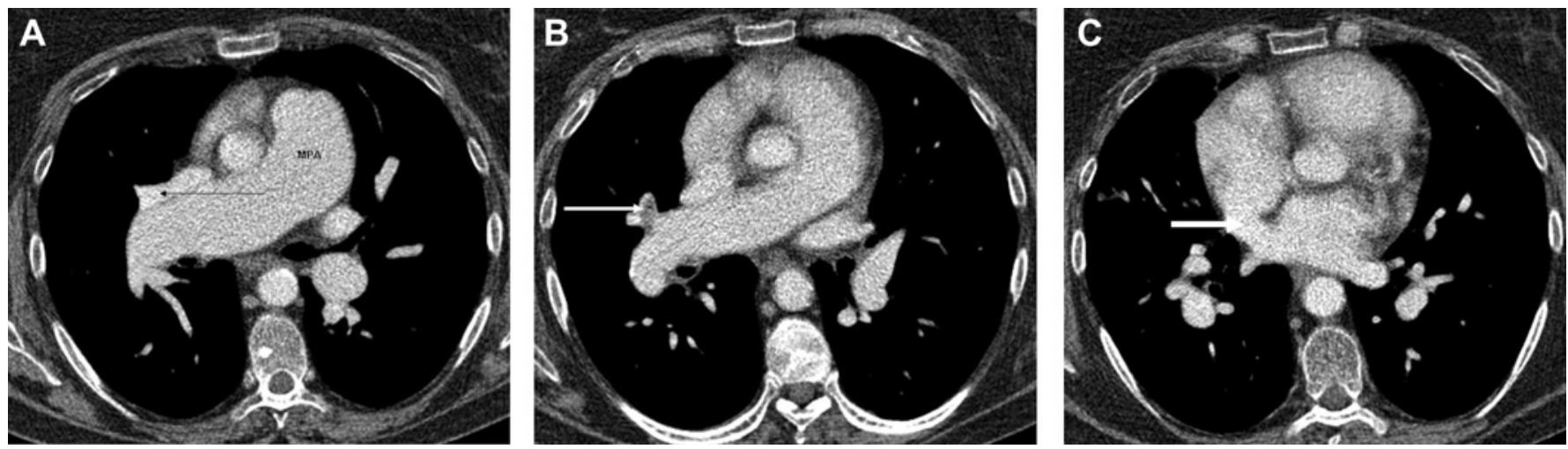

Figure 8 Contrast-enhanced pulmonary angiography in a 67-year-old female patient with pulmonary hypertension demonstrating congenital abnormalities and acute pulmonary embolus. (A) Dilated main pulmonary artery (MPA) with anomalous pulmonary venous drainage; note a right upper lobe vein draining into the superior vena cava (arrow). (B) Non-occlusive thrombus in the right middle lobe lobar artery and the medial segmental branch (block arrow). (C) Communication between the atria in keeping with a sinus venosus type atrial septal defect (block arrow).

myocardial mass to be assessed for either ventricle in the shortaxis or transverse plane. Despite suggestion that the inter-study reproducibility of the $\mathrm{RV}$ is less than for the $L V,{ }^{33} \mathrm{CMR}$ is still considered a reliable method for the assessment of RV morphology and function, ${ }^{34}$ with a large RV volume and a low stroke volume being independent predictors of mortality and treatment failure. ${ }^{35}$ Right ventricular end-diastolic wall thickness has been shown to correlate well with MPAP in IPAH and some cases of secondary PAH. ${ }^{36} 37$ A linear relationship has also been described between right ventricular mass and MPAP in IPAH (figure 9).$^{38}$ Cine images also demonstrate the dynamic distortion of the normal shape of the interventricular septum. With raised right ventricular pressures, the interventricular septum flattens and bows towards the left ventricle, with systolic pressures being proportional to septal curvature in patients with $\mathrm{PH}^{39}$ Right ventricular delayed hyper-enhancement can be identified in patients with $\mathrm{PH}$ and has been shown to correlate with right

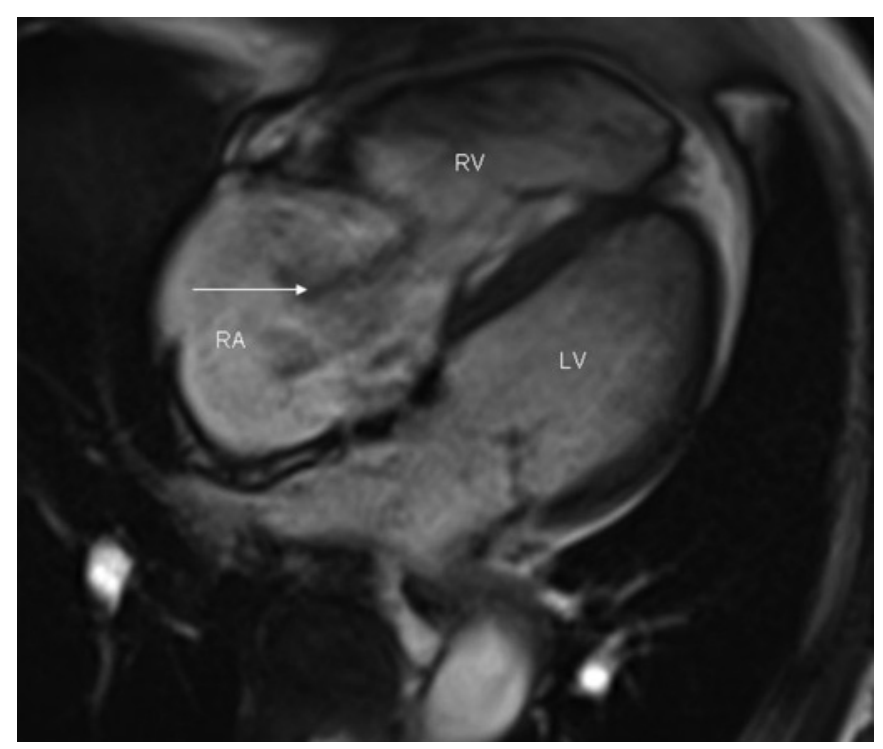

Figure 9 Four-chamber view from a steady state free precision technique cardiac magnetic resonance sequence in a 29 -year-old female patient with chronic thromboembolic pulmonary hypertension. There are dilated right heart chambers and a jet of signal void across the tricuspid valve extending into the right atrium (arrow) representing tricuspid regurgitation. LV, left ventricle; $\mathrm{RA}$, right atrium; RV, right ventricle. ventricular dysfunction. ${ }^{40}$ The enhancement pattern involves the RV septal insertion points and the interventricular septum with septal enhancement being associated with septal bowing. ${ }^{41}$ However, delayed enhancement in the RV insertion point is a non-specific sign and can be seen in many disease entities and some normal subjects as well.

The pulmonary circulation can be assessed visually by threedimensional gadolinium magnetic resonance angiography and quantified using velocity-encoded cine imaging. Magnetic resonance angiography demonstrates good correlation with conventional angiography and CTPA. ${ }^{42} 43$ Velocity-encoded imaging produces a map of cross-sectional flow in a vessel over time. In patients with $\mathrm{PAH}$, findings include decreased pulmonary artery distensibility, increased retrograde flow and inhomogeneous velocity profiles. $^{44}$ Sanz et al showed a strong correlation between the blood flow velocity in the pulmonary artery and the severity of $\mathrm{PAH}$, suggesting that a decrease in pulmonary blood flow velocity is proportional to the increase of pulmonary vascular resistance. ${ }^{45}$ A cut-off value of $11.7 \mathrm{~cm} / \mathrm{s}$ was established to differentiate between patients with and without PAH. Another finding that can be demonstrated on MRI phase contrast sequences is reduced systolic right coronary artery blood flow. In PAH, the peak and mean systolic flows are reduced compared with the diastolic flow. This is due to compression of the right coronary artery by the hypertrophied $\mathrm{RV}$ and is directly related to $\mathrm{RV}$ mass. ${ }^{46}$

\section{RIGHT HEART CATHETERISATION AND CATHETER PULMONARY ANGIOGRAPHY}

Right heart catheterisation is required to confirm the diagnosis and pulmonary angiography may also be performed at the same time. It allows assessment of pulmonary haemodynamics, cardiac function and vasoreactivity of the pulmonary circulation. Although invasive, when performed at experienced centres, the morbidity and mortality are low $(1.1 \%$ and $0.05 \%$, respectively). ${ }^{47}$ Multiple variables are recorded including the right atrial and ventricular pressures, mean, systolic and diastolic PAP, and cardiac output. Pulmonary capillary wedge pressure (or the left ventricular end diastolic pressure) is vital to exclude $\mathrm{PH}$ due to left heart disease. Oxygen saturation recording is performed to look for shunting as a possible aetiology.

Vasoreactivity testing should be performed to identify patients who may benefit from long term therapy with calcium channel blockers. The agent most commonly used is inhaled 
Figure 10 Catheter pulmonary angiogram in a 56 -year-old patient with chronic thromboembolic pulmonary hypertension. (A) A completely occluded left lower lobe artery (black arrow). (B) Proximal complex web in right lower lobe artery (white arrow)
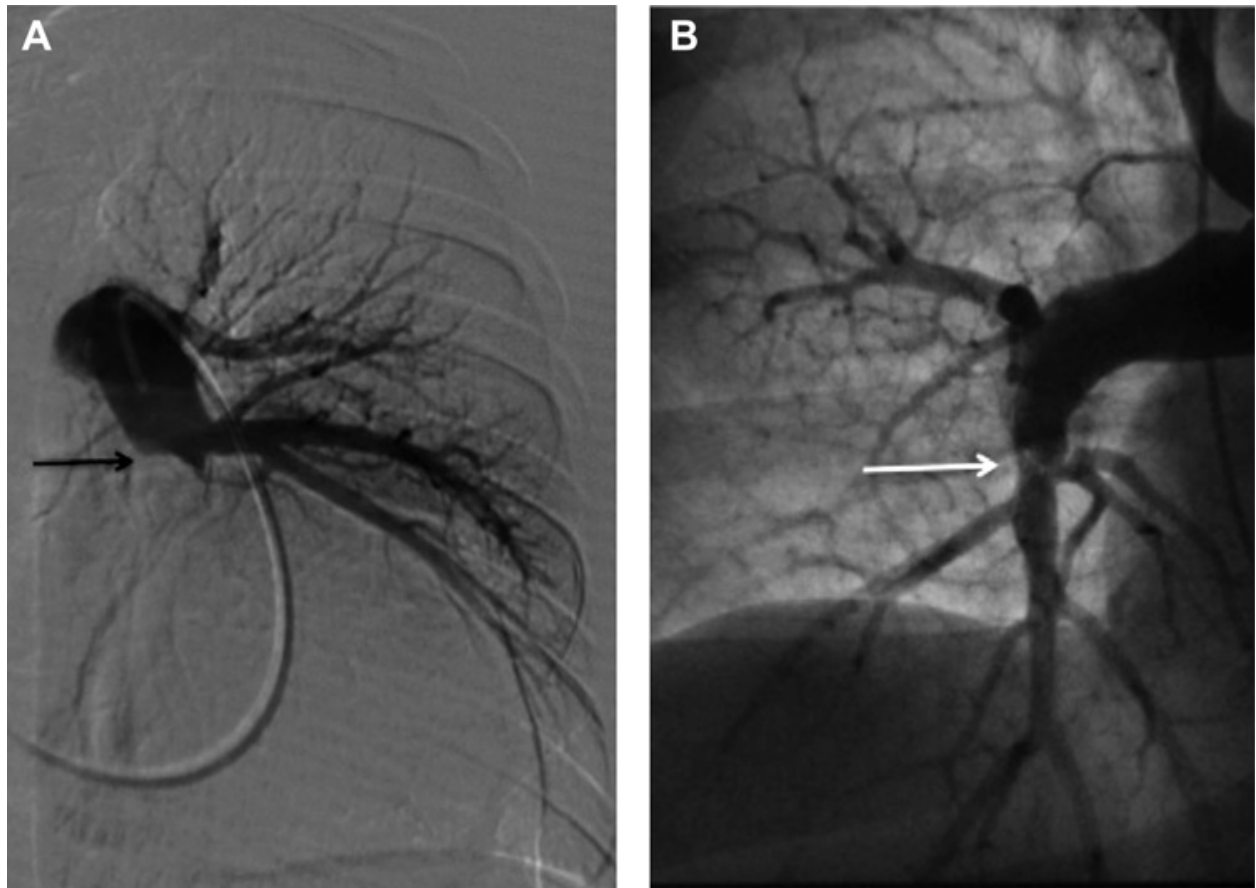

nitric oxide. ${ }^{48}$ A positive response is a reduction in the MPAP of $>10 \mathrm{~mm} \mathrm{Hg}$ to a value of $40 \mathrm{~mm} \mathrm{Hg}$ or less, with an increased or unchanged cardiac output. Only about $10 \%$ of IPAH patients meet these criteria with about half of these being positive long term responders to calcium channel blockers. Pulmonary angiography is the gold standard technique for defining the anatomy of the pulmonary circulation. It is most useful in the assessment of chronic thromboembolic disease defining the extent and assessing the suitability for surgical intervention (figure 10). It has excellent temporal resolution and is less hampered by motion artefact and venous contamination that is generated with CT and CMR. However, the latter modalities are becoming increasing popular due to their non-invasiveness, ready availability and provision of additional extra-luminal morphologic and/or functional information. In the future, conventional angiography will probably only be performed if an adequate roadmap of the pulmonary vasculature has not been provided by CT or CMR. ${ }^{49}$

\section{IMAGING ALGORITHM}

Equipment availability, expertise and cost are some key factors that determine the choice of various imaging modalities in the investigation of $\mathrm{PH}$. The protocol given in figure 11 has been shown to be effective and reliable at our institution.
Figure 11 Imaging algorithm for $\mathrm{PH}$ used in our institution. CTEPH, chronic thromboembolic pulmonary hypertension; CTPA, contrast-enhanced pulmonary angiography; IPAH, hypertension; V/Q, ventilation/perfusion. idiopathic pulmonary arterial hypertension; $\mathrm{PH}$, pulmonary

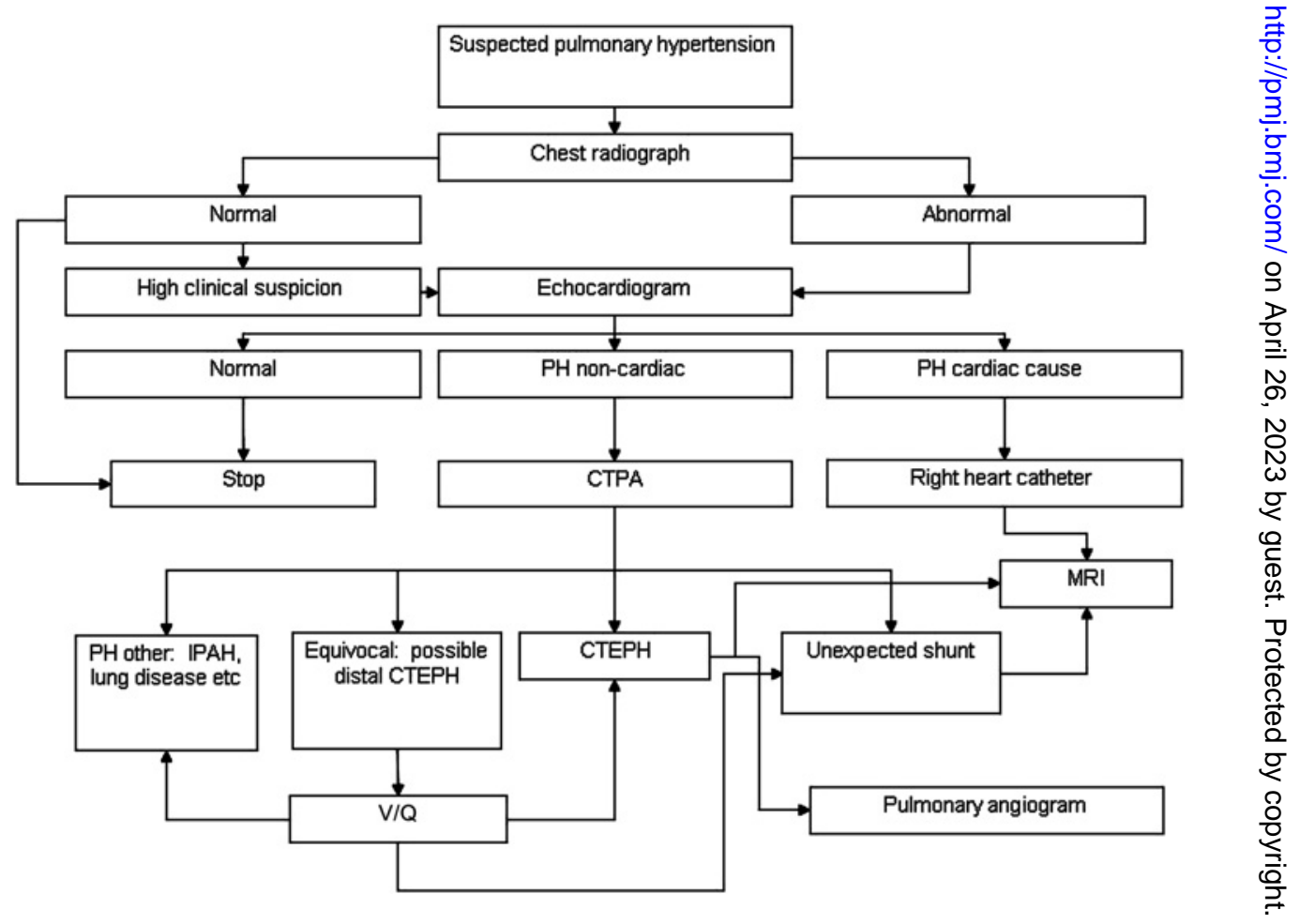




\section{Main messages}

- Presenting symptoms of pulmonary hypertension (PH) are often non-specific leading to a delay in diagnosis averaging about 2 years or indeed misdiagnosis.

- Echo is a great tool to screen for patients at risk of PH but can miss mild PH particularly if there is no tricuspid regurgitation.

- Generic imaging features of PH include cardiomegaly, right atrial and right ventricular dilatation, and enlarged central pulmonary arteries.

- Ventilation/perfusion scintigram is mainly performed to differentiate chronic thromboembolic pulmonary hypertension from other causes of pulmonary arterial hypertension.

- Vasoreactivity testing should be performed during right heart catheterisation to identify patients who may benefit from long term therapy with calcium channel blockers.

- Although pulmonary angiography is the gold standard technique for defining the anatomy of the pulmonary circulation, non-invasive techniques such as MR pulmonary angiography are emerging as an acceptable alternative with the added attraction of being able to provide additional extraluminal morphologic and functional information.

All suspected patients start with a chest radiograph. If it is abnormal or if there is a high clinical suspicion of PAH based on history, examination, ECG and pulmonary function test, an echocardiography is performed. If the echocardiogram is normal, no further imaging is necessary but if abnormal or equivocal, further investigation is required. If echo features are suggestive of $\mathrm{PAH}$ but no non-cardiac cause is found on echo, then a combined CTPA and HRCT is performed. Any further imaging depends on the CTPA findings. When an occult intracardiac shunt is identified, CMR will be helpful for shunt quantification and ventricular functional analysis. Equivocal CT findings are followed by nuclear scintigraphy to exclude distal thromboembolic disease. If there is proximal CTEPH, additional imaging using MR pulmonary angiography is performed to further define the anatomy and disease extent for the surgeon. Where $M R$ is contraindicated, catheter pulmonary angiography is used as an alternative. All patients have right heart catheterisation for confirming the diagnosis and assessing disease severity. Follow-up post-pulmonary endarterectomy is performed using right heart catheterisation, CTPA and CMR. IPAH is a diagnosis of exclusion.

\section{CONCLUSION}

$\mathrm{PAH}$ is uncommon but associated with significant morbidity and mortality. It may present late with non-specific signs and

\section{Current research questions}

- Can Magnetic Resonance (MR) pulmonary angiography replace invasive catheter pulmonary angiography as the gold standard for morphological assessment of pulmonary vasculature?

- Is it feasible to reliably measure pulmonary vascular resistance using non-invasive imaging such as Cardiac Magnetic Resonance?

- What is the best way of evaluating patients suspected of having exercise-induced pulmonary hypertension?

\section{Key references}

Galie N, Hoeper MM, Humbert M, et al. Guidelines for the diagnosis and treatment of pulmonary hypertension. Eur Respir J 2009;34:1219-63.

- Simonneau G, Robbins IM, Beghetti M, et al. Updated clinical classification of pulmonary hypertension. J Am Coll Cardiol 2009;54:S43-54.

- Muller N, Fraser RS, Colman N, et al. Radiologic Diagnosis of Diseases of the Chest. Philadelphia: WB Saunders, 2001.

- Oudkerk M, van Beek EJ, Wielopolski P, et al. Comparison of contrast-enhanced magnetic resonance angiography and conventional pulmonary angiography for the diagnosis of pulmonary embolism: a prospective study. Lancet 2002;359:1643-7.

- Coulden R. State-of-the-art imaging techniques in chronic thromboembolic pulmonary hypertension. Proc Am Thorac Soc 2006;3:577-83.

symptoms. Imaging plays a pivotal role by identifying specific causes, quantifying disease severity, as well as helping in directing treatment. Since cross-sectional imaging is commonly performed in patients with non-specific cardiorespiratory symptoms, it is important that imagers and clinicians routinely consider whether there are imaging features suggestive of $\mathrm{PH}$. The various modalities play complementary roles in assessment with precise imaging algorithm varying according to local facilities and expertise.

\section{SELF ASSESSMENT QUESTIONS (TRUE/FALSE; ANSWERS AFTER THE REFERENCES)}

1. $\mathrm{PH}$ is defined as systolic pulmonary arterial pressure of $>25 \mathrm{~mm} \mathrm{Hg}$ at rest.

2. The size of the pulmonary arterial enlargement on chest radiography correlates well with the severity of $\mathrm{PAH}$.

3. $V / Q$ scintigraphy has high sensitivity and specificity in distinguishing between CTEPH and IPAH.

4. Pulmonary arterial dilatation can be seen on CT in the absence of $\mathrm{PH}$.

5. Catheter pulmonary angiography has better temporal resolution compared with MRI.

Funding This work was supported by the NIHR Cambridge Biomedical Research Centre.

\section{Competing interests None.}

Contributors CM: First author, researched and wrote the paper. DG: Corresponding author, researched and co-wrote the paper and did the images. KS: Clinician, contributed to writing clinical aspects of the manuscript. NS: Senior author, undertook overall review.

Provenance and peer review Commissioned; externally peer reviewed.

\section{REFERENCES}

1. Hatano S, Strasser T. Primary Pulmonary Hypertension. Geneva: World Health Organisation, 1975

2. Kovacs G, Berghold A, Scheidl S, et al. Pulmonary arterial pressure during rest and exercise in healthy subjects: a systematic review. Eur Respir J 2009:34:888-94.

3. Badesch BD, Champion HC, Gomez-Sanchez MA, et al. Diagnosis and assessment of pulmonary hypertension. J Am Coll Cardiol 2009;54:S55-6.

4. Naeije R, Melot C, Niset G, et al. Mechanisms of improved arterial oxygenation after peripheral chemoreceptor stimulation during hypoxic exercise. J Appl Physiol 1993;74:1666-71.

5. Galie N, Hoeper MM, Humbert M, et al. Guidelines for the diagnosis and treatment of pulmonary hypertension. Eur Respir J 2009;34:1219-63. 
6. Peacock AJ. Treatment of pulmonary hypertension. BMJ 2003;326:835-6.

7. D'Alonzo GE, Barst RJ, Ayres SM, et al. Survival in patients with primary pulmonary hypertension. Results from a national prospective registry. Ann Intern Med 1991:115:343-9.

8. Rich S, Rubin IJ, Abenhaim L, et al. Executive Summary From the World Symposium on Primary Pulmonary Hypertension. Evian, France: World Health Organisation, 1998.

9. Simonneau G, Galie N, Rubin IJ, et al. Clinical classification of pulmonary hypertension. J Am Coll Cardiol 2004;43:5S-12S.

10. Simonneau G, Robbins IM, Beghetti M, et al. Updated clinical classification of pulmonary hypertension. J Am Coll Cardiol 2009;54:S43-54.

11. Machado RD, Aldred MA, James V, et al. Mutations of the TGF-beta type II receptor BMPR2 in pulmonary arterial hypertension. Hum Mutat 2006;27:121-32.

12. Machado R, Eickelberg 0, Elliott CG, et al. Genetics and genomics of pulmonary arterial hypertension. J Am Coll Cardiol 2009;54:S32-42.

13. Chaouat A, Coulet F, Favre $C$, et al. Endoglin germline mutation in patient with hereditary haemorrhagic telangiectasia and dexfenfluramine associated pulmonary arterial hypertension. Thorax 2004;59:446-8.

14. Chaves E. The pathology of the arterial pulmonary vasculature in Manson's schistosomiasis. Chest 1966;50:72-7.

15. Haque AK, Gokhale S, Rampy BA, et al. Pulmonary hypertension in sickle cell haemoglobinopthy: a clinicopathological study of 20 cases. Hum Pathol 2002;33:1037-43.

16. Rich S, Dantzker DR, Ayres SM, et al. Primary pulmonary hypertension. A national prospective study. Ann Intern Med 1987:107:216-23.

17. Chang $\mathbf{C H}$. The normal roentgenographic measurement of the right descending pulmonary artery in 1085 cases. Am J Roentgenol Radium Ther Nucl Med 1962;87:929-35.

18. Muller N, Fraser RS, Colman N, et al. Radiologic Diagnosis of Diseases of the Chest. Philadelphia: WB Saunders, 2001.

19. Gutierrez FR, Moran CJ, Ludbrook PA, et al. Pulmonary arterial calcification with reversible pulmonary hypertension. Am J Roentgenol 1980;135:177-8

20. Fisher FR, Forfia PR, Chamera E, et al. Accuracy of doppler echocardiography in the haemodynamic assessment of pulmonary hypertension. Am J Resp Crit Care Med 2009;179:615-21.

21. Fedullo PF, Auger WR, Channick RN, et al. Chronic thromboembolic pulmonary hypertension. Clin Chest Med 2001;22:561-81.

22. Tunariu N, Gibbs SJR, Win Z, et al. Ventilation-perfusion scintigraphy is more sensitive than multidetector CTPA in detecting chronic thromboembolic pulmonary disease as a treatable cause of pulmonary hypertension. J Nucl Med 2007;48:680-4.

23. Kuriyama K, Gamsu G, Stern RG, et al. CT-determined pulmonary artery diameters in predicting pulmonary hypertension. Invest Radiol 1984;19:16-22.

24. Ng CS, Wells AU, Padley SP. A CT sign of chronic pulmonary arterial hypertension: the ratio of main pulmonary artery to aortic diameter. $J$ Thorac Imaging 1999;14:270-8

25. Devaraj A, Wells AU, Meister MG, et al. Detection of pulmonary hypertension with multidetector CT and echocardiography alone and in combination. Radiology 2010;254:609-16

26. Devaraj A, Wells AU, Meister MG, et al. The effect of diffuse pulmonary fibrosis on the reliability of CT signs of pulmonary hypertension. Radiology 2008;249:1042-9.

27. Tan RT, Kuzo R, Goodman LR, et al. Utility of CT scan evaluation for predicting pulmonary hypertension in patients with parenchymal lung disease. Medical college of Wisconsin lung transplant group. Chest 1998;113:1250-6.

28. Groves AM, Win T, Charman SC, et al. Semi-quantitative assessment of tricuspid regurgitation on contrast-enhanced multi-detector CT. Clin Radiol 2004;59:715-19.

29. Perez-Enguix D, Morales P, Tomas JM, et al. Computed tomographic screening of pulmonary arterial hypertension in candidates for lung transplantation. Transplant Proc 2007:39:2405-8.

30. Quiroz R, Kucher N, Schoepf UJ, et al. Right ventricular enlargement on chest computed tomography: prognostic role in acute pulmonary embolus. Circulation 2004;109:2401-4

31. Stein PD, Matta F, Yaekoub AY, et al. Reconstructed 4-chamber views compared with axial imaging for assessment of right ventricular enlargement on CT pulmonary angiograms. J Thromb Thrombolysis 2009;28:342-7.

32. Resten A, Maitre S, Humbert M, et al. Pulmonary arterial hypertension: thin section CT predictors of epoprostenol therapy failure. Radiology 2002;222:782-8.
33. Grothues F, Moon JC, Bellenger NG, et al. Interstudy reproducibility of right ventricular volumes, function, and mass with cardiovascular magnetic resonance $A m$ Heart J 2004;147:218-23.

34. Boxt LM, Katz J, Kolb T, et al. Direct quantitation of right and left ventricular volumes with nuclear magnetic resonance imaging in patients with primary pulmonary hypertension. J Am Coll Cardiol 1992:19:1508-15.

35. Van Wolferen SA, Marcus JT, Boonstra A, et al. Prognostic value of right ventricular mass, volume, and function in idiopathic pulmonary arterial hypertension. Eur Heart J 2007:28:1250-7.

36. Bouchard A, Higgins CB, Byrd BF 3rd, et al. Magnetic resonance imaging in pulmonary arterial hypertension. Am J Cardiol 1985;56:938-42.

37. Saito H, Dambara T, Aiba M, et al. Evaluation of cor pulmonale on a modifed shortaxis section of the heart by magnetic resonance imaging. Am Rev Respir Dis 1992;146:1576-81.

38. Katz J, Whang J, Boxt LM, et al. Estimation of right ventricular mass in normal subjects and in patients with primary pulmonary hypertension by nuclear magnetic resonance imaging. J Am Coll Cardiol 1993:21:1475-81.

39. Roeleveld RJ, Marcus JT, Faes TJ, et al. Interventricular septal configuration at MR imaging and pulmonary arterial pressure in pulmonary hypertension. Radiology 2005:234:710-17.

40. Blyth KG, Groenning BA, Martin TN, et al. Contrast-enhanced cardiovascular magnetic resonance imaging in patients with pulmonary hypertension. Eur Heart $J$ 2005;26:1993-9.

41. McCann GP, Gan CT, Beek AM, et al. Extent of MRI delayed enhancement of myocardial mass is related to right ventricular dysfunction in pulmonary artery hypertension. Am J Roengentol 2007:188:349-55.

42. Oudkerk M, van Beek EJ, Wielopolski $\mathrm{P}$, et al. Comparison of contrast-enhanced magnetic resonance angiography and conventional pulmonary angiography for the diagnosis of pulmonary embolism: a prospective study. Lancet 2002;359:1643-7.

43. Roche KJ, Rivera R, Argilla $\mathrm{M}$, et al. Assessment of vasculature using combined MRI and MR angiography. Am J Roentgenol 2004;182:861-6

44. Kondo C, Caputo GR, Masui T, et al. Pulmonary hypertension: pulmonary flow quantification and flow profile analysis with velocity encoded cine MR imaging. Radiology 1992;183:751-8.

45. Sanz J, Kuschnir P, Rius T, et al. Pulmonary hypertension: noninvasive detection with phase contrast MR imaging. Radiology 2007;243:70-9.

46. Van Wolferen S, Marcus JT, Westerhof N, et al. Right coronary flow impairment in patients with pulmonary hypertension. Eur Heart J 2008;29:120-7.

47. Hoeper MM, Lee SH, Voswinckel R, et al. Complications of right heart catheter procedures in patients with pulmonary hypertension in experienced centres. J Am Coll Cardiol 2006;48:2546-52.

48. Sitbon 0, Humbert $M$, Jais $X$, et al. Long-term response to calcium channel blockers in idiopathic pulmonary arterial hypertension. Circulation 2005:111:3105-11.

49. Coulden R. State-of-the-art imaging techniques in chronic thromboembolic pulmonary hypertension. Proc Am Thorac Soc 2006;3:577-83.

\section{ANSWERS}

1. False: $\mathrm{PH}$ is defined as mean pulmonary arterial pressure of $>25 \mathrm{~mm} \mathrm{Hg}$ at rest.

2. False: The size of the pulmonary artery on radiography does not correlate well with haemodynamic severity of PAH.

3. True: V/0 sensitivity is $90 \%-100 \%$ and specificity is $94 \%-100 \%$ in distinguishing these two conditions.

4. True: Pulmonary arterial dilatation can be seen in patients with pulmonary fibrosis without $\mathrm{PH}$.

5. True: Pulmonary angiography remains the gold standard for delineation of the pulmonary vasculature although it is invasive and does not give any functional information. 